\title{
Haemorrhagic Lupus: A Diagnosis Emergency, Report about 6 Cases
}

\author{
Fall Biram Codou', Walah Dimitri', Dieng Mouhamed ${ }^{2}$, Diaw Bamba1, Tode Johanita ${ }^{1}$, \\ Gaye Ahmadou', Salane Aly1, Ndao Awa Cheikh², Cisse Amadou Fall'3, Sow Maimouna², \\ M. A. Ndour1', Djiba Boundia², Kane Baidy Sy², Fall Seynabou ${ }^{4}$, Leye Abdoulaye ${ }^{3}$, \\ Pouye Abdoulaye ${ }^{2}$, Ndongo Souhaibou ${ }^{1}$
}

\author{
${ }^{1}$ Department of Internal Medicine at DalalJamm Hospital, Dakar, Sénégal \\ ${ }^{2}$ Department of Internal Medicine at Dantec Hospital, Dakar, Sénégal \\ ${ }^{3}$ Department of Internal Medicine at Pikine Hospital, Dakar, Sénégal \\ ${ }^{4}$ Department of Hematology at DalalJamm Hospital, Dakar, Sénégal \\ Email: biramfall86@gmail.com
}

How to cite this paper: Codou, F.B., Dimitri, W., Mouhamed, D., Bamba, D., Johanita, T., Ahmadou, G., Aly, S., Cheikh, N.A., Fall, C.A., Maimouna, S., Ndour, M.A., Boundia, D., Sy, K.B., Seynabou, F., Abdoulaye, L., Abdoulaye, P. and Souhaibou, N. (2021) Haemorrhagic Lupus: A Diagnosis Emergency, Report about 6 Cases. Open Journal of Internal Medicine, 11, 231-237. https://doi.org/10.4236/ojim.2021.114019

Received: April 9, 2021

Accepted: December 7, 2021

Published: December 10, 2021

Copyright ( 2021 by author(s) and Scientific Research Publishing Inc. This work is licensed under the Creative Commons Attribution International License (CC BY 4.0).

http://creativecommons.org/licenses/by/4.0/ (c) (i) Open Access

\begin{abstract}
Introduction: Haemorrhagic manifestations rarely represent the mode of onset of lupus and are a therapeutical and diagnosis emergency. We report 6 cases of haemorrhagic manifestations disclosing lupus. Patients and methods: It's about a retrospective, descriptive and multicentric study ranged from 1st January 2014 to 5th January 2019. Were included in the study all cases of lupic disease revealed by hemorrhagic disorders, recorded in 3 Internal Medicine Departments in Dakar: DalalJamm, Dantec, Pikine. The diagnosis of lupus was made on the basis of the 1997 modified classification criteria of ACR for SLE. Results: We gathered 6 cases of women affected by lupic disease. The mean age was 32 years old with extremes ranged from 17 to 56 years. According to haemorrhagic disorders, the diagnosis of lupus was automatically made in 5 of the cases and it took 6 months to confirm the last one. Haemorrhagic features were ruled by purpura (3/6 cases) and nosebleeding associated with gumbleeding ( $2 / 6$ cases), epistaxis and melena (1/6 case), hypermenorrhea $(1 / 6$ case), cerebral haematoma (1/6case). Cutaneous (5/6), articular (4/6), renal (2/6) and serous (2/6) disorders specially represented the other manifestations. Biological findings showed cytopenia with 3 cases of anaemia, 1 case of leucopenia and 6 cases of thrombopenia. The average of the platelet count was at 20,000 cells $/ \mathrm{mm}^{3}$ with extremes ranged from 1000 to 35,000 cells $/ \mathrm{mm}^{3}$. Thrombocytopenia was related to autoimmune thrombocytopenic purpura in 3 cases, to Evans' syndrome in 2 cases, to macrophage activation syndrome in 1 case. Immunological investigations found the presence of antinuclear antibodies (1/6), anti-dsDNA (2/6) and anti-Sm (4/6). All patients received a bolus of Solumedrol followed by an administration of Prednisone at a dosage of
\end{abstract}


$1.5 \mathrm{mg} / \mathrm{kg}$ (3 cases) and $1 \mathrm{mg} / \mathrm{kg}$ to the other patients. A transfusion of platelet concentrates was performed for all of them. The evolution was favorable in 4 cases. Two patients died according to a septic and haemorrhagic shock. Conclusion: We report 6 cases of haemorrhagic manifestations disclosing lupus. Cutaneo-mucous location was more frequent and was related to moderate to severe thrombocytopenia with the presence of anti-Sm antibodies.

\section{Keywords}

Haemorrhagic, Lupus, Emergency

\section{Introduction}

Lupus is a systemic autoimmune disease with a broad spectrum of clinical presentations evolving by flare-ups and remissions [1] [2]. It is more common on young females. Estimated incidence rates in USA and Europe range $1-23$ per 100,000 per year. Prevalence in adults is as high as 150 per 100,000 in United States and is 20 - 50 per 100,000 in Europe [3] [4]. Its etiology is unclear but many observations suggest a role of genetic and environmental factors such as immunologic, hormonal factors, physical factors. It has variable mode of onset ruled by cutaneous, articular and hematological disorders [3] [4]. Other uncommon and life-threatening manifestations can reveal the disease such as hemorrhagic features. There are several reports of hemorrhagic features. Li J. et al. reported $8 \%$ of hemorrhagic cases in their study [5]. We report 6 cases of lupic disease disclosed by a hemorrhagic syndrome.

\section{Materials and Methods}

It's about a retrospective, descriptive and multicentric study range from 1st January 2014 to 5th January 2019. Were included in the study all cases of lupic disease revealed by hemorrhagic disorders, recorded in 3 Internal Medicine Departments in Dakar: DalalJamm, Dantec, Pikine. The diagnosis of lupus was made on the basis of the 1997 modified classification criteria of ACR. If the patients had at least 4 criteria on 11 then they were classified as a Lupus. The data analysis was made with Microsoft excel 2007 and SPSS version 20.

\section{Results}

We gathered 6 cases of women affected by lupic disease. The mean age was 32 years old with extremes range 17 and 56 years. According to hemorrhagic disorders, the diagnosis of lupus was automatically made in 5 of the cases and it took 6 months to confirm the last one. Hemorrhagic features were ruled by purpura ( 3 cases) and nosebleeding associated to gumbleeding ( 2 cases) (Table 1). Cutaneous (5/6) and articular (4/6) disorders specially represented the other manifestations. Biological findings showed cytopenia with 3 cases of anaemia, 1 case of leucopenia and 6 cases of thrombopenia (Table 2). 
Table 1. Haemorrhagic patterns during admission.

\begin{tabular}{cc}
\hline Haemorrhagic manifestations & Number of cases \\
\hline Purpura & 3 \\
Epistaxis associated with gingivorragia & 2 \\
Epistaxis associated with melena & 1 \\
Hypermenorrhea & 1 \\
Cerebral haematoma & 1 \\
\hline
\end{tabular}

Table 2. Clinical and biological features during the diagnostic of lupus.

\begin{tabular}{cc}
\hline Clinical and biological manifestations & Number of cases \\
\hline Cutaneous manifestations & 5 \\
Articular manifestations & 4 \\
Renal manifestations & 2 \\
Serous manifestations & 2 \\
Anemia & 3 \\
leucopenia & 1 \\
Thrombocytopenia & 6 \\
Antinuclear antibodies & 1 \\
Anti-dsDNA antibodies & 2 \\
Anti-Sm antibodies & 4 \\
\hline
\end{tabular}

The average of the platelet count was at 20,000 cells $/ \mathrm{mm}^{3}$ with extremes range from 1000 to 35,000 cells $/ \mathrm{mm}^{3}$. Thrombocytopenia was related to autoimmune thrombocytopenic purpura in 3 cases, to Evans syndrome in 2 cases, to macrophage activation syndrome in 1 case (Figure 1). Immunological investigations found the presence of antinuclear antibodies in 1 patient, anti-dsDNA in 2 patients and anti-Sm in 4 patients (Table 2). All the patients received a bolus of Solumedrol followed by an administration of Prednisone at a dosage of 1.5 $\mathrm{mg} / \mathrm{kg}$ ( 3 cases) and $1 \mathrm{mg} / \mathrm{kg}$ to the other patients. A transfusion of platelet concentrates was performed for all of them. They were under Azathioprine and Hydroxychloroquine respectively at a dosage of $100 \mathrm{mg} /$ day and $400 \mathrm{mg} /$ day, after having a normal platelet count. The evolution was favorable in 4 cases. Two patients died according to a septic and haemorrhagic shock.

\section{Discussion}

We conducted a study on inaugural hemorrhagic manifestations that lead to the diagnosis of lupic disease.

Articular, cutaneous and hematologic features are the leading modes of onset of lupic disease [3] [4]. Hematologic disorders of the lupus are common at early stage or at the time of diagnosis. Up to date, hemorrhagic onset is rare as we can note with the rarity of medical reports in our level [6] [7] [8]. Li J and coll noted 


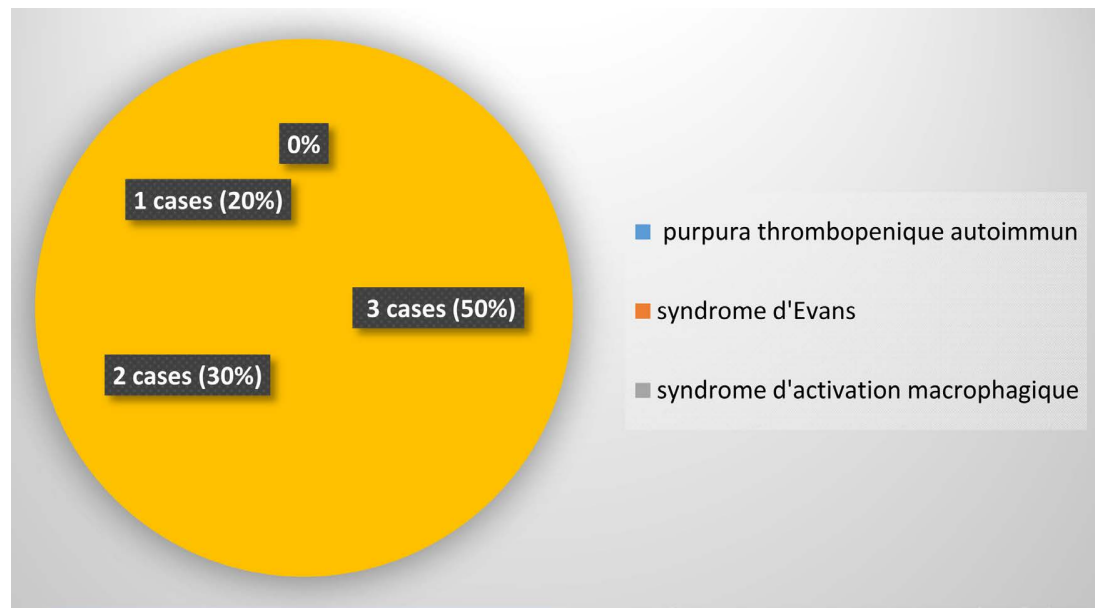

Figure 1. Mechanism of thrombocytopenia.

hemorrhagic disorders during lupus at a frequency of $8 \%$ while Jallouli and coll found a frequency of 6\% [5] [9]. In 2010 in Senegal, Fatou Samba and coll reported 2 cases of hemorrhagic manifestations that to say $1.4 \%$ in her series [10]. We report 6 cases of hemorrhagic manifestations revealing the lupic disease.

The occurrence of hemorrhagic manifestations during lupic disease is a diagnostic and therapeutic emergency, as we could see in our study where we had 5 patients on whom the diagnosis of lupic disease was made during hospitalization.

Lupic disease is a condition that mostly occurs in women at their thirthies [3] [4]. This inequality is due to hormonal factors involvement especially ostrogens by a stimulation of the immune system. Hemorrhagic manifestations are not excepted as shown in many studies [5] [9] [11]. In our study, the mean age was estimated at 32 years with extremes range 17 and 56 years old. Li and coll found in their study in regard to thrombocytopenia during lupus with hemorrhagic features, an average age of $34.8 \pm 14.6$ years proving the early onset of the condition and its hallmarks [5].

In our study, purpura, epistaxis and gingivorragia constituted the main hemorrhagic manifestations. These data are similar to those reported on the literature [5] [6] [11]. Li J. and coll found that $70 \%$ of bleeding patients with severe thrombocytopenia $\left(<20,000\right.$ platelets $\left./ \mathrm{mm}^{3}\right)$ and $75 \%$ with moderate thrombocytopenia had cutaneo-mucous involvement. According to that, how severe the thrombocytopenia could be, the predilection of hemorrhage would be cutaneo-mucous.

At the time of diagnosis, the main manifestations were articular and cutaneous. That was similar to the findings according to the literature where the manifestations occur simultaneously to the initial clinical course [5] [9] [11].

The thrombocytopenia occurring during lupic disease is due to a lack of production of the bone marrow, a splenic sequestration or a rapid peripheral destruction related to the autoimmune process. Commonly, that's a mild low platelet count [12]. Hemorrhagic features mostly occur with moderate (20.000 50.000 cells $/ \mathrm{mm}^{3}$ ) to severe (less than 20.000 cells $/ \mathrm{mm}^{3}$ ) thrombocytopenia [5] 
[9] [11]. Li J. and coll found that $76 \%$ of people with severe low platelet count underwent a hemorrhage against $25 \%$ patients with moderate low platelet count [5]. This leads to say the lower the platelet count is, the higher the risk of bleeding. In Tunisia, Jallouli and coll found similar datas [9]. This is proven in our study with a mean platelet count of 20.000 cells $/ \mathrm{mm}^{3}$ with extremes range of 1000 and 50,000 cells $/ \mathrm{mm}^{3}$.

These low platelet levels were mostly associated with idiopathic autoimmune thrombocytopenia, antiphospholipid syndrome, Evans' syndrome. Autoimmune thrombocytopenia related to lupus is defined by a platelet count less than 100.000 cells $/ \mathrm{mm}^{3}$ without another underlying cause. It occurs in $7 \%-30 \%$ cases of lupus. Antiphospholipid syndrome is a condition featuring thrombotic manifestations, obstetrical accidents and antiphospholipid antibodies. Evans syndrome is characterized by the association of autoimmune haemolytic anaemia and thrombocytopenia [12]. An association of thrombocytopenia with Evans syndrome was found in $12.5 \%$ by Tokano and coll, in $1.2 \%$ by Li and coll and 18\% by Ziakas and coll [5] [11] [13]. Li and coll found an association of thrombocytopenia with antiphospholipid syndrome in $6 \%$ of cases while Jallouli and coll found $14.3 \%$ cases. In our study, thrombocytopenia was associated with autoimmune thrombopenic purpura in 50\% of cases, in 33\% with an Evans syndrome and $17 \%$ with macrophage activation syndrome. The different findings could be explained by a selection bias in our study. Because all our patients presented haemorrhagic manifestations with thrombocytopenia. However, the studies were conducted on people having thrombocytopenia with or without haemorrhagic manifestations.

The diagnosis of lupic disease is confirmed on the basis of biological and immunological findings especially the dosage of fluorescent antinuclear antibodies. Further investigations can be performed for more specific antibodies such as anti-dsDNA and anti-Sm. The series provided by Jallouli and coll showed a positivity of anti-dsDNA antibodies in $73.5 \%$ and anti-Sm in $32.4 \%$ respectively. The one conducted by Jung and coll showed a positivity of $100 \%$ of anti-DNA and $8.3 \%$ of anti-Sm on patients with thrombocytopenia. Ziakas and coll reported, respectively, a positivity of $100 \%$ for antinuclear antibodies, $56 \%$ for anti-DNA and $10 \%$ for anti-Sm [9] [11] [14]. In our series, the presence of antinuclear antibodies were noted in $17 \%$ of the cases, while anti-DNA and anti-Sm were respectively found in $33 \%$ and $60 \%$ of the cases. The different findings could be explained by the ethnical and racial difference in our studied populations. In fact, Meyer and coll noticed a sensibility of anti-Sm antibodies of $30 \%$ on African Americans and $3 \%$ to $7 \%$ in european series. Anti-Sm sensibility is especially high on black people with a proportion of $50 \%$ compared to what is seen in Caucasians on whom we note a proportion of $10 \%$ to $20 \%$ of SLE cases [15].

The urgent management of autoimmune thrombocytopenia or secondary haemorrhagic manifestations during lupus requires high doses of glucocorticoids. A second-line treatment may consist on the administration of immunosuppressors such as azathioprine and cyclophosphamide. In case the aforemen- 
tioned molecules don't work, rituximab can be used as a third-line treatment. Transfusion of concentrates platelets should be performed only if the platelet count is less than 10.000 cells $/ \mathrm{mm}^{3}$ [12]. In our study, the patients received a bolus of methylprednisolone followed by prednisone at a dosage of $1.5 \mathrm{mg} / \mathrm{kg}$ and $1 \mathrm{mg} / \mathrm{kg}$. After normalizing the platelet count, patients were under azathioprine at a dosage of $100 \mathrm{mg} /$ day and hydroxychloroquine at a dosage of 400 $\mathrm{mg} /$ day. All patients underwent a transfusion of concentrates platelets.

After treatment, the evolution was favorable in 4 patients. Two patients died after due to haemorrhagic and septic shock. This was similar to the datas reported on the literature. Central neurological disorders and infections constitute the leading mortality causes after inaugural haemorrhage. Li J and coll reported a mortality rate of $12.1 \%$ on bleeding patients. They also found a mortality rate estimated at $13 \%$ on patients with mild to severe thrombocytopenia as a leading cause. Infections and hemorrhagic shock respectively caused $49 \%$ and $33 \%$ of deaths [5] [9] [11] [14]. This points out the importance of an early diagnosis and management of these manifestations.

The limitation of our study is the small sample of our study. That is not enough to take real statement and tendency on clinical and paraclinical findings that appeared with an initial hemorrhagic manifestation that lead to the lupus' diagnosis.

\section{Conclusion}

We report 6 cases of Lupus revealed by hemorrhagic disorders. Mostly, the bleeding areas are cutaneous and mucous associated with mild to severe thrombocytopenia related to autoimmune thrombocytopenic purpura. They occur with the presence of anti-Sm antibodies. A study conducted on a larger sample would be necessary, on black African people, to point out the prevalence of hemorrhagic manifestations during systemic lupus and its pathogenesis.

\section{Conflicts of Interest}

The authors declare no conflicts of interest regarding the publication of this paper.

\section{References}

[1] Meyer, O. and Kahn, M.-F. (2000) Lupus érythémateux systémique. In: Maladies et syndromes systémiques, 4e Edition, Flammarion, Paris, 131-368.

[2] Subra, J.F., Druet, P. and Fournie, G.J. (2000) Physiopathologie des maladies lupiques. Médecine Thérapeutique, 6, 529-536.

[3] Faezi, T.M., Almodarresi, M.H., Akbarian, M., et al. (2014) Clinical and Immunological Pattern of Systemic Lupus Erythematosus in Men in a Cohort of $2355 \mathrm{~Pa}$ tients. International Journal of Rheumatic Diseases, 17, 394-399. https://doi.org/10.1111/1756-185X.12268

[4] Batool, S., Ahmad, N.M., Saeed, M.A., et al. (2016) Pattern of Initial Clinical Manifestations of Systemic Lupus Erythematosus in a Tertiary Care Hospital. Pakistan 
Journal of Medical Sciences, 32, 1066-1070. https://doi.org/10.12669/pims.325.11480

[5] Li, J., Pan, Z., Liu, H., et al. (2018) Retrospective Analysis of the Risk of Hemorrhage Associated with Moderate and Severe Thrombocytopenia of 173 Patients with Systemic Lupus Erythematosus. Medicine, 97, e11356. https://doi.org/10.1097/MD.0000000000011356

[6] Pons-Estel, G.J., Ugarte-Gil, M.F. and Alarcón, G.S. (2017) Epidemiology of Systemic Lupus Erythematosus. Expert Review of Clinical Immunology, 13, 799-814. https://doi.org/10.1080/1744666X.2017.1327352

[7] Kallingal, M.P. (2010) SLE Presenting as Epistaxis. Indian Journal of Pediatrics, 77, 561-562. https://doi.org/10.1007/s12098-010-0069-2

[8] Ratti, N., Cypierre, A., Bézanahary, H., et al. (2017) Un lupus qui saigne. La Revue de médecine interne, 38S, A110-A225. https://doi.org/10.1016/j.revmed.2017.10.013

[9] Jallouli, M., Frigui, M., Marzouk, S., et al. (2012) Clinical Implications and Prognostic Significance of Thrombocytopenia in Tunisian Patients with Systemic Lupus Erythematosus. Lupus, 21, 682-687. https://doi.org/10.1177/0961203312438630

[10] Ndiaye, F.S.D., Ka, M.M., Fall, S., et al. (2010) Les manifestations hématologiques et immunologiques de la maladie lupique: L'expérience du centre hospitalo-universitaire de Dakar. Hématologie, 16, 318-323. https://doi.org/10.1684/hma.2010.0439

[11] Ziakas, P.D., Giannouli, S., Zintzaras, E., et al. (2005) Lupus Thrombocytopenia: Clinical Implications and Prognostic Significance. Annals of the Rheumatic Disease Journal, 64, 1366-1369. https://doi.org/10.1136/ard.2004.033100

[12] Velo-García, A., Castro, S.G. and Isenberg, D.A. (2016) The Diagnosis and Management of the Haematologic Manifestations of Lupus. Journal of Autoimmunity, 74, 139-160. https://doi.org/10.1016/j.jaut.2016.07.001

[13] Tokano, Y., Morimoto, S., Amano, H., et al. (2005) The Relationship between Initial Clinical Manifestation and Long-Term Prognosis of Patients with Systemic Lupus Erythematosus. Modern Rheumatology, 15, 275-282. https://doi.org/10.3109/s10165-005-0411-0

[14] Jung, J.-H., Soh, M.-S., Ahn, Y.-H., et al. (2016) Thrombocytopenia in Systemic Lupus Erythematosus Clinical Manifestations, Treatment, and Prognosis in $230 \mathrm{~Pa}-$ tients. Medicine, 95, e2818. https://doi.org/10.1097/MD.0000000000002818

[15] Haddouk, S., Ben Ayed, M., Baklouti, S., et al. (2005) Autoanticorps dans le lupus érythémateux systémique: Profil et corrélations cliniques. Pathologie Biologie, 53, 311-317. https://doi.org/10.1016/j.patbio.2004.10.004 